\title{
EL ROL DE LA UNIUERSIDAD EN LA PRODUCCIÓN SOCIAL DEL HÁBITAT: INERCIAS Y DESAFIOS
}

María J. KISZKA

mariajosekiszka@hotmail.com

Sec. de Extensión y Transferencia FAU-UNNE. Prof. Adj. de Tecnología Gráfica I (Diseño Gráfico). Maestranda en Antropología Social (UNAM) y en Gestión y Desarrollo de la Vivienda Social (UNNE).

Palabras Clave: Universitaria; producción social del hábitat; gestión del conocimiento.

Keywords: university extension; social production of habitat; knowledge management

\section{RESUMEN}

El presente trabajo tiene como objetivo problematizar el rol social de la universidad en la producción social del hábitat desde la Facultad de Arquitectura y Urbanismo (UNNE). Para ello aborda las normativas y encuadres de trabajos vigentes, en pos de construir lineamientos de trabajo adecuados a las efectivas demandas. Desde la interacción de las políticas y las efectivas prácticas se busca dilucidar las lógicas que tienden a reforzar lo instituido en términos de prácticas y cuáles son las que propician escenarios efectivos de trabajo con la comunidad.

\section{ABSTRACT}

This paper aims to problematize the social role of the University in the social production of habitat from the Faculty of Architecture and Urbanism (UNNE). For this, it addresses the rules and frames of current works, after building work guidelines appropriate to the effective demands. From the interaction between policies and effective practices, seeks to elucidate the logic that tend to reinforce what was instituted in terms of practices and what are the scenarios that promote effective work with the community. 


\section{OBJETIVOS}

\section{OBJETIVO GENERAL}

Problematizar el rol social de la universidad en la producción social del hábitat desde la facultad de Arquitectura y Urbanismo

\section{OBJETIVOS PARTICULARES}

- Indagar sobre el estado del arte de la política de extensión universitaria en sus diversas escalas: universidad, facultad, institutos y cátedras.

- Caracterizar los escenarios y prácticas en los que la universidad cumple su rol social, para materializar un diagnóstico.

\section{INTRODUCCIÓN}

El presente trabajo expone discusiones vigentes en el seno de la política universitaria, desde su rol social, en particular desde la extensión universitaria, poniendo énfasis en el acontecer de la Facultad de Arquitectura y Urbanismo de la UNNE. Aborda reflexivamente las estrategias de los distintos sectores, que en carácter de agentes, operan en este escenario. Se concibe que la interacción entre los lineamientos políticos y las efectivas prácticas ofrece una disparidad, por lo que se busca dilucidar las lógicas que tienden a reforzar las acciones instituidas en términos de prácticas y cuáles son las necesarias para propiciar escenarios efectivos de trabajo con la comunidad. Por ello se problematizan aquí las normativas y encuadres de trabajos vigentes, con la intención de construir lineamientos de trabajo adecuados a las efectivas demandas.
Se propone aportar al desarrollo del concepto de Producción Social del Hábitat desde una perspectiva integral, para luego reflexionar sobre posibles estrategias de abordaje, desde el encuadre universitario. Se tienen por objeto diversas experiencias desarrolladas desde la universidad y como aporte a la PSH, de distintas áreas de la región en la que se inserta la UNNE'. Estos avances se constituyen en insumos para definir lineamientos de trabajo para una propuesta que incida en los procesos de producción social del hábitat desde las prácticas de extensión de la FAU, en el marco de proyectos y desarrollo curricular.

\section{El rol social de la universidad}

El Estatuto de la Universidad Nacional del Nordeste, en su artículo primero, enuncia como fin prioritario de la institución "la generación y comunicación de conocimientos del más alto nivel, y la formación ética, cultural, técnica y profesional, contribuyendo al esclarecimiento de los problemas y necesidades de todo orden de la región, para beneficio del hombre y extendiendo su acción y servicios a la Comunidad a la que pertenece, en un clima de libertad, justicia, igualdad y solidaridad" 2 . A continuación, señala que no debe desintegrar los problemas sociales, sino que debe estudiarlos científicamente. En este sentido, uno de los primeros obstáculos expuestos — que aún no se ha podido superar - es la carencia de encuadres que permitan la incidencia significativa de estos

\footnotetext{
1 Esta investigación exploratoria se vincula con el desarrollo de dos tesis de maestría en curso: "El rol de la Universidad en la Producción social del hábitat" y "Caracterización del hábitat QOM en contexto Urbano". El presente trabajo, además, se enriquece a la luz de los desarrollos producidos en el marco del proyecto de investigación "Hábitat y Desigualdad Social. Antropología de las áreas urbanas deficitarias críticas (AUDC) en el AMGR", cuya directora es María Andrea Benítez, radicado en el Instituto de Investigación y desarrollo en Vivienda (IIDVi) de la FAU-UNNE, desde donde abordo la problemática de población indígena en territorio. 2 Fuente: Estatuto de la Universidad Nacional del Nordeste.
} 
"...se problematizan aquí las normativas y encuadres de trabajos vigentes, con la intención de construir lineamientos de trabajo adecuados a las efectivas demandas."

propósitos en lo cotidiano, en los trayectos o en la vida profesional de la comunidad académica.

Toda política debe traducirse en herramientas concretas y apropiables por los intérpretes y destinatarios, y en estos términos la gestión es la herramienta. En Argentina nos encontramos universidades que como parte de la currícula cuentan con cátedras de Extensión, en las cuales se articulan las diversas disciplinas; también se reconocen diversas cátedras que trabajan con las problemáticas de las comunidades en las que se encuentran insertas. Pero estos escenarios de ninguna manera generan condiciones de transformación contundente, son más bien definibles como aportes o experiencias y no de acciones integrales por diversas causas.

El mencionado estatuto expone en su artículo ciento cuatro que el proceso educativo se erige de manera sistémica y solidaria promoviendo al hombre y su entorno, como agente de colaboración y progreso. Nos habla de un sujeto de formación sistémica con compromiso social, pero en el análisis de las estructuras y las prácticas cotidianas, este cometido no se refleja: programas de extensión que no inciden ni en el $3 \%$ de participación de los agentes de la gran mayoría de las distintas facultades, escasos espacios de interacción entre facultades y entre áreas del conocimiento, elitización de la investigación cuando debería formar parte de la currícula cotidiana y no como un modo de especialización dentro de la profesión, que además se disocia de los espacios de docencia y extensión.

La comunidad académica de la UNNE, integrada por alumnos, docentes, investigadores, personal no docente y graduados, en la negociación cotidiana entre sus ideales y sus prácticas resuelve sus contradicciones exponiendo en cada uno de estos roles, como principal cuestión la promoción de capacidades y valores autónomos. En el artículo segundo del estatuto se manifiesta la intención de "formar integral e interdisciplinariamente a sus componentes e infundir en ellos el espíritu crítico y a la vez el sentido de responsabilidad personal y social de reafirmación ético cívica y de sus convicciones democráticas y republicanas" 3 . Se destaca como principio el reconocimiento de que los diversos enfoques son reconocidos y considerados como tales promoviendo la construcción a partir del disenso. La contradicción sustancial entre lo que se expone y una evaluación general de las prácticas concretas se manifiesta en aspectos presupuestarios; cantidad y calidad de espacios de vinculación con la comunidad; modalidades de cursado y promoción de los estudiantes; el sistema de exigencias y valoraciones para la carrera docente y de investigadores; la carencia de presupuesto, encuadre y valoración de la dimensión de la extensión universitaria y el desmerecimiento del aporte del personal no docente en cada una de las dimensiones de trabajo.

DE SOUSA SANTOS (2012) entiende una nueva forma de pensar la vinculación de la universidad con el medio a través de la ecología de saberes, y propone trabajos vinculados con el medio, generados desde demandas externas hacia el seno de la Academia: "Consiste en la promoción de diálogos entre el saber científico y humanístico que la universidad produce y los saberes legos, populares, tradicionales, urbanos, campesinos, provincianos, de cultura no occidentales (indígenas, de origen africano, oriental, etc.) que circulan en la sociedad" (DE SOUSA SANTOS, 2012: 172). Esta mirada nos permitiría recuperar construcciones culturales particulares de la región, ponderarlas y elevarlas a instancias superiores para la generación de herramientas más apropiadas, en la construcción de saberes, en camino hacia lo que hoy se considera como justicia cognitiva.

3 Fuente: Estatuto de la Universidad Nacional del Nordeste. 
Según un informe del Centro de Estudios de la Educación Argentina (CEA), que depende de la Universidad de Belgrano, el Estado invierte anualmente en sus alumnos un promedio de \$ 7777, y un graduado tiene un costo estimado en $\$ 133.196$ en la Universidad Nacional del Nordeste ${ }^{4}$. Asimismo, en una reciente nota publicada, DARÍO MIZRAHI señala: "en Argentina los pobres financian el estudio de los ricos. Mientras el grueso de los que se reciben son de sectores medios y altos, no hay becas para que los de clase baja puedan dedicarse sólo a estudiar"5; al respecto el Estado argentino a partir de 2014 se traza una política de financiación para los jóvenes de dieciocho a veinticuatro años denominada Progresar, destinada a una ayuda económica que permita a los jóvenes de bajos recursos posibilitar su educación. Estos datos, a la luz de que por año egresan 2,5 profesionales cada mil habitantes, dan cuenta de la imperiosa necesidad de que la influencia de transformación de un profesional argentino sea plena y absoluta. ¿Qué políticas de optimización de recursos vinculados con la incidencia en la transformación del medio se dan con los profesionales formados?

El rol del profesional es el de facilitador, sea docente, investigador o un profesional que ejerce de manera liberal. Se presenta en escena como una herramienta que permite acercar propuestas a las demandas y donde el rol de la comunidad no es el de espectadora, consumidora o destinataria, más bien debería plantearse en términos de agencia. Asimismo, existe en la actualidad un problema de enfoque, ya que las prácticas deben orientarse a la producción de trabajos que permitan a las comunidades generar sus propios dispositivos, una vez concluido el trabajo académico, como

\footnotetext{
4 Fuente: Boletín informativo N. ${ }^{\circ} 5$ del Centro de Estudios de la Educación Argentina de la Universidad de Belgrano. http://184.168.109.199:8080/jspui/bitstream/123456789/1259/1/ cea_numero_5.pdf.

5 http://www.infobae.com/2013/06/08/714525-el-estado-invierte-200-mil-pesos-ano-cada-graduado-universitario.
}

se dice habitualmente: trabajos orientados al empoderamiento comunitario. Reconocer y ponderar los espacios de producción colectiva del conocimiento es tarea de todos. Pensar un espacio de trabajo universidad-comunidad implica el reconocimiento de capacidades y saberes de ambos sectores, accesibilidad, construcción de un lenguaje. En este sentido, el gran desafío de la universidad es pensarse como agente de cambio, sin perder la mirada crítica, el rol de construcción de conocimiento, de la legitimidad de sus saberes, pero con prácticas más concretas que la simple reflexión: acción y reflexión para no caminar a ciegas.

El dilema vigente es que los tiempos de la universidad son muy lentos y las demandas muy urgentes, y así se estereotipa el divorcio del pensar sin actuar y el actuar sin reflexionar. La extensión dentro de la formación no debiera ser una opción, así como la integración de los contenidos o las prácticas de diversas problemáticas si se piensa en la producción de profesionales al servicio del desarrollo de la comunidad. De igual manera, la investigación no debería estar tan divorciada de la mayoría de las prácticas curriculares. Los debates se dan en lo cotidiano, y se expone como contradicción algo que debería verse como dialógico y dialéctico, como un capital sinérgico de apropiación de la realidad para transformarla. El gran desafío de la extensión universitaria es no limitarse a las propuestas de financiación que ofrecen diversos programas, y partir del ejercicio cotidiano de trabajo integrado en lo curricular, en lo formativo y en la generación de conciencia de profesionales comprometidos con el medio al que se aspiran a insertar.

\section{La extensión universitaria como posibilidad de aprendizaje y transformación}

DE SOUSA SANTOS (2012) reflexiona respecto del encuadre capitalista que tiñe el funcionamiento del sistema mundo; la Universidad teje como alternativa una empresa cuyo bien de cambio 
es el conocimiento, al servicio de este sistema, proponiendo en estos términos una necesaria "reforma de la universidad [que]debe conferir una nueva centralidad a las actividades de extensión (con implicaciones en el currículo y en las carreras de los docentes) y concebirlas de modo alternativo al capitalismo global, atribuyendo a las universidades una participación activa en la construcción de la cohesión social, en la profundización de la democracia en la lucha contra la exclusión social, la degradación ambiental y en la defensa de la diversidad cultural" (DE SOUSA SANTOS 2012: 169).

Actualmente la extensión se vincula con gestiones que se deben realizar en el marco de trabajos desarrollados por investigadores, docentes y alumnos. La pregunta que cabe es ¿cuántos de estos agentes de la comunidad universitaria realizan sus trabajos vinculados con las necesidades del medio de manera efectiva? A efectos del análisis vamos a desarrollar una de las dimensiones en cuestión para el abordaje de la producción social del hábitat, que se entiende como encuadre de trabajo principal: la Extensión Universitaria. A tal efecto de presentarán programas, implementaciones y resultados.

El programa Universidad en el Medio promueve el desarrollo de Proyectos de Extensión transformadores de la realidad social, económica y productiva de la región, tendientes a mejorar la calidad de vida, optimizar el uso de los recursos productivos y a la adecuada gestión y el desarrollo integral sustentable de los diferentes sectores de la comunidad. Los equipos de trabajo tendrán carácter interdisciplinario, integrados por docentes de la UNNE y estudiantes universitarios que hayan aprobado por lo menos la mitad de las materias del plan de estudios de la carrera que cursan. Podrán participar personal no docente de la UNNE y organismos e instituciones provinciales, nacionales e internacio- nales públicas o privadas ${ }^{6}$. Alrededor de cincuenta proyectos por año abordan diversas problemáticas de la región, pero el impacto es muy bajo si consideramos que la UNNE abarca a la comunidad del Chaco y Corrientes y que el número de agentes implicados no supera el $1 \%$ del total de la población universitaria.

El programa de "Voluntariado Universitario" desarrollado por la Dirección Nacional de Desarrollo Universitario y Voluntariado del Ministerio de Educación de la Nación se inscribe dentro de un proyecto político que considera que la Educación Superior es prioritaria para el crecimiento de un país en todos sus aspectos, y que por ello debe ser un medio para la producción de conocimiento y su redistribución a toda la sociedad. "Tiene como objetivo general profundizar la función social de la Universidad, integrando el conocimiento generado en las aulas con las problemáticas más urgentes de nuestro país. Así, se busca hacer un aporte para que las actividades realizadas por los estudiantes y docentes estén orientadas a trabajar junto a la comunidad. Forma parte de las políticas del Estado en materia educativa que buscan fortalecer este proyecto de país inclusivo y solidario. Con esta impronta, se propone generar un diálogo real entre la Universidad y el Pueblo, con la participación de escuelas, organizaciones sociales y la comunidad en general junto a los estudiantes universitarios y docentes, con el fin de trabajar colectivamente en la planificación y logro de objetivos comunes, que no pueden ser otros que los de la mayoría"7.

Estas líneas de crédito y trabajo tienen un porcentaje de incidencia muy bajo si se considera la población universitaria y las urgentes demandas de la comunidad. Existen además convocatorias de proyectos de diversos organismos públicos a nivel nacional, de carácter extraordinario. Un programa

6 Fuente: http://www.unne.edu.ar/entorno/unne_enelmedio.php

7 Fuente: http://portales.educacion.gov.ar/spu/voluntariado-universitario/. 
en vías de consolidación es el de la Secretaría de Políticas Universitarias, en cuya $15 .^{\text {a }}$ Convocatoria de extensión universitaria "Universidad, estado y territorio", se seleccionaron a nivel país 270 proyectos financiados de un total de 727 propuestas presentadas. Se destinarán 10 millones de pesos para cubrir estos proyectos, lo que da cuenta de la apuesta del Ministerio de Educación por revalorizar la función de la Extensión Universitaria. Esta convocatoria pretende articular a la universidad con su entorno social, multiplicando los vínculos con los diferentes actores e instituciones para dar respuestas concretas y eficaces a problemáticas sociales, socio-productivas y culturales ${ }^{8}$. Estos esfuerzos por fuera de lo estructural de la práctica cotidiana universitaria, como opción o elección, refuerzan la idea de que la extensión está ligada al asistencialismo, al voluntarismo, que trata de cubrir la veta noble y sensible de los ciudadanos, perdiendo de vista la riqueza de la extensión como herramienta de transformación social.

En los diversos escenarios del cotidiano en la vida universitaria, se disocia el acto de construir conocimiento del acontecer del entorno inmediato, edificando saberes desde abstracciones, considerando muy poco la materialización de acciones y su impacto en el medio. Los motivos son diversos: falta de financiamiento, falta de aprovechamiento de los recursos humanos, carencia de encuadres apropiados, entre los más verbalizados. En este sentido, la investigación recurso altamente formativo - transita en una práctica de alto provecho para un número muy reducido de agentes. Suele aparecer en alguna cátedra, aislada de la currícula o como una especialización de la vida profesional y no como una dimensión de verificación entre realidadteoría-práctica, como instrumento de reflexión y producción del conocimiento.

8 Fuente: http://portales.educacion.gov.ar/spu/extension-y-vinculacion-tecnologica.
Una corriente emergente que va ganando territorio es la investigación-acción, cuyas actividades identifican estrategias de acción que son implementadas y más tarde sometidas a observación, reflexión y cambio. Se considera como un instrumento que genera cambio social y conocimiento sobre la realidad, instalando capacidades a quienes la realizan. En términos de DA SOUSA SANTOS, investigación-acción "consiste en la definición y ejecución participativa de proyectos de investigación, involucrando a las comunidades y a las organizaciones sociales populares en la medida en que la solución de los problemas les puede beneficiar como resultado de la investigación. Los intereses sociales están articulados con los intereses científicos de los investigadores y la producción del conocimiento científico se da estrechamente ligada a la satisfacción de necesidades de los grupos sociales que no tienen poder para poner el conocimiento técnico y especializado a su servicio a través de la vía mercantil" (DE SOUSA SANTOS, 2012: 171). De esta conceptualización cabe destacar la integralidad, intersectorialidad y visión sistémica de las acciones.

En un reciente libro, una experiencia realizada en el seno de la Universidad de Córdoba, en la cátedra que aborda la problemática de la vivienda social, FERRERO Y REBORD (2013) plantean que en el desarrollo concreto de más de veinte años de trayectoria, desde el año 1991, "la Universidad resulta referencial, marcando rumbos con respecto a que busca una sociedad cuando forma a sus ciudadanos; la educación superior se entiende, no como mera instancia que favorece la salida laboral o el fortalecimiento de la lógicas profesionalistas del mercado, sino como ámbito de desarrollo de la capacidad de pensamiento crítico; como patrimonio común de la sociedad toda, trasciende la formación científico-académica y debe orientarse en pos de la acción transformadora hacia sociedades más justas y equitativas. En definitiva, una sociedad responsable de la universidad de la cual se nutre 
"Una corriente emergente que va ganando territorio es la investigación-acción, cuyas acti-vidades identifican estrategias de acción que son implementadas y más tarde sometidas a observación, reflexión y cambio."

y una universidad comprometida con la sociedad que la contiene" (FERRERO Y REBORD, 2012: 53). Estos términos, consideramos, como principios son muy buenos, aplicados en una cátedra son mejores, pero nos cabe reflexionar sobre cuáles son los términos que debieran considerarse para que gran parte de la estructura académica se brinde de hecho a abordar las problemáticas de la comunidad. Entiendo que el retraso en esta materia no se debe al desconocimiento o a la falta de voluntad; la complejidad que estos términos requieren implica un cambio de paradigma aún en proceso desde las gestiones políticas y académicas que rigen nuestras universidades.

\section{La extensión universitaria desde la facultad de arquitectura y urbanismo de la unne}

El concepto de hábitat refiere al conjunto de bienes y servicios que configuran posiciones, identidades y prácticas de sujetos y grupos sociales, en un entramado socio-territorial más amplio. El territorio es la construcción social del espacio, es el resultado de las relaciones sociales especializadas; más precisamente: son las relaciones de poder espacialmente delimitadas. En consecuencia, se entiende como territorialidad al conjunto de prácticas y sus expresiones materiales y simbólicas (RODRÍGUEZ, SCABUZZO ET ÁL. 2013: 18).

Un abordaje simplista — como al que muchas veces se recurre desde las operatorias del Estado y las organizaciones - erige propuestas indigentes en cuanto al impacto de transformación de las actuales condiciones de la ciudadanía. Disputar el sentido de estas prácticas es un desafío mayor que el que podemos comprender: es de posicionamiento frente a la problemática. La intervención desde una perspectiva compleja demanda entonces innovar, generar mecanismos flexibles, abiertos y dinámicos partiendo desde la mirada del desarrollo local y utilizando las herramientas que brinda, entre otras disciplinas, la Psicología Social, para el óptimo impulso de trabajo, en el que se reconozcan procesos más que resultados y roles más que actores clave. Es por ello fundamental comenzar a visualizar horizontes que promuevan la articulación e interacción entre la multiplicidad de actores que abordan la problemática del hábitat. La universidad, cuyo aporte se traduce en insumos de gestión para las comunidades y organismos, es un actor cuya presencia se aspira a ponderar en este trabajo.

Para ser rigurosos en el desarrollo de este concepto debemos apelar a su infinitud dada por la multiplicidad de factores: cada ser en cada medio del que proviene, o no, en cada acción e interacción produce y re-produce su hábitat. Dicha interacción va dejando huellas; cada marca preestablecida es vuelta a modificar con otra acción grande o pequeña a cada instante. Aspirar a realizar una interpretación de un fenómeno tiene que poder ser algo más que tomar una fotografía o en el mejor de los casos visualizar de qué va la película; tiene que permitirnos teatralizar, en el sentido de estar próximos a la escena que transcurre.

La Facultad de Arquitectura y Urbanismo cuenta con escasos trabajos de impacto significativo que aborden problemáticas urgentes del medio. Estando en una de las provincias más pobres de la Argentina, su vinculación con ellas es más que estratégica: es necesaria. Este desperdicio de recursos humanos y horas de trabajo es plenamente mensurable si consideramos las siguientes variables: cantidad de docentes, investigadores y alumnos, multiplicados por sus horas de trabajo y desempeño del rol en la comunidad académica y contabilizamos cuántos de estos productos/producidos hoy salen en los diarios o están transformando en términos de desarrollo, en el medio en el que se insertan. Los desafíos que se plantean en cuanto a extensión son el reconocimiento curricular de las prácticas, que debiera adaptarse a procesos de docentes y alumnos, sin perder de vista las demandas del medio, la generación de nuevos espacios, entre otras líneas de trabajo. 


\section{La producción social del hábitat}

La comprensión actual de la Producción Social del Hábitat (PSH) implica la intervención de múltiples disciplinas, por lo que abordarla de manera integral enlaza a las diversas ramas del conocimiento dialécticamente. Es considerada intersectorial, ya que políticos, instituciones, comunidad y otros agentes expresan intereses y manejan diversos espacios de poder. Por la falta de herramientas para la comprensión, todo se sigue viendo como una sumatoria de las partes y no desde la integración. El origen principal de este fenómeno es la formación que se recibe como estudiantes universitarios, que repercute luego en el ejercicio profesional.

La visión presente de la PSH tiene diversos matices que es necesario exponer, a fines de clarificar. Al respecto GUSTAVO ROMERO expone que en la década del 70 se han admitido enfoques que van desde la interpretación como "proceso de desarrollo evolutivo", fenómeno que abordaría las carencias habitacionales de sectores que son excluidos, y enfoques como el de Enrique Ortiz Flores, quien parte de reconocer la iniciativa de distintos tipos de organizaciones que llevan la vanguardia de la gestión social en la producción de viviendas y conjuntos habitacionales (ROMERO, 2002: 75). Para ser rigurosos en el desarrollo del concepto de PSH, debemos apelar a su infinitud dada por la multiplicidad de factores: cada ser en cada medio del que proviene, en cada acción e interacción produce y re-produce su hábitat. Dicha interacción va dejando huellas donde cada marca preestablecida es vuelta a modificar con otra acción grande o pequeña a cada instante. Aspirar a realizar una interpretación de un fenómeno tiene que poder ser algo más que tomar una fotografía o en el mejor de los casos visualizar de qué va la película, tiene que permitirnos teatralizar, en el sentido de estar próximos a la escena que transcurre.

ROMERO plantea entonces como una posible definición de PSH a un "sistema que permite que los individuos, las familias, las comunidades y las diferentes organizaciones sociales produzcan vivienda y hábitat en forma tal que controlen las decisiones fundamentales, ya sea en forma individual o en conjunto, mediante procesos que tiendan a evolucionar hacia formas más complejas y efectivas. Ello implica que deben existir la políticas, las estrategias, los instrumentos, la legislación, la normatividad, los financiamientos, las asesorías $y$, en fin, los diferentes caminos, posibilidades y opciones que lo faciliten" (ROMERO, 2002: 77). Esta definición reconoce una necesaria mirada integral de la problemática habitacional en nuestras ciudades, que en su mayoría se construyen desde la informalidad. Si tomamos como caso de estudio en el Área Metropolitana del Gran Resistencia (en adelante AMGR), en donde se incide, la producción del suelo manifiesta que las ocupaciones de territorio van configurando, vemos fenómenos que escapan a los desairados intentos de previsión y planificación del Estado.

Por lo general, la problemática del hábitat responde a distintos factores que es bueno considerar. En diversas experiencias de campo, se pudo constatar que las intervenciones del Estado en materia de vivienda realizan diagnósticos de distintas escalas de rigor, planifican, intervienen y se retiran. Por otro lado, se invisibilizan los procesos previos que han realizado las instituciones locales (escuela, centro de salud, iglesia, entre las más destacadas), y son las que forman parte de la historia y trascienden a los gobiernos, gestiones e intervenciones, siendo esta la oportunidad precisa de transformación. Desde esta perspectiva es bueno reconocer el rol que ejercen en terreno los educadores, los médicos, los religiosos. 
"...la PSH se presenta de manera multiactoral, multisectorial, multi-dimensional y en permanente proceso de transformación."

Por otra parte, la vivienda como objeto no representa de manera absoluta el espacio de reproducción de la vida cotidiana, y es bueno reconocer en este sentido la importancia de verificación de funcionamientos y adaptación de los espacios recreativos y las instituciones implicadas en el proceso, entre una larga lista de requerimientos y aspectos que resolver. En la operación de la PSH conviene tener presente lo planteado por RODRÍGUEZ, SCABUZZO Y OTROS, quienes señalan que "el concepto de hábitat refiere al conjunto de bienes y servicios que configuran posiciones, identidades y prácticas de sujetos y grupos sociales, en un entramado socio-territorial más amplio. El territorio es la construcción social del espacio, es el resultado de las relaciones sociales especializadas, más precisamente son las relaciones de poder espacialmente delimitadas, en consecuencia se entiende territorialidad al conjunto de prácticas y sus expresiones materiales y simbólicas" (RODRÍGUEZ, SCABUZZO ET ÁL., 2013: 18).

En términos de FREIRE, de "encuentro de saberes", o al decir de PICHÓN RIVIERE, de "enseñajes", instancia en donde el que enseña aprende y el que aprende enseña, se hace necesario reflexionar sobre nuevos paradigmas de producción social del hábitat. El reconocimiento de capacidades, acciones, aptitudes, caminos y obstáculos, resistencias, prácticas cotidianas, entre otros capitales para la transformación, genera umbrales de conocimiento y acción más efectivos. A efectos de abordar este enfoque, se pone a consideración el aporte de ENET (2008) en su publicación "Hábitat Evolutivo", quien propone como modalidad de trabajo el Gerenciamiento Participativo Sustentable, definido como "la construcción intersectorial de un proyecto y o programa negociado y concertado en un proceso continuo de planificación estratégica flexible y descentralizada, apta para encontrar alternativas adecuadas en un contexto de complejidad e incertidumbre y desarrollar propuestas más equitativas basadas en la participación efectiva" (ENET, 2008: 19).

Para ello explican los autores que es necesario un proceso de fortalecimiento de los actores implicados, entiendo que no desde una nivelación, sino más bien de una construcción dialéctica del lenguaje. Esto implica romper con ciertos paradigmas de trabajo e instituir prácticas que ya desde la planificación propenden al fortalecimiento comunitario.

Otro aporte de ENET (2008) en esta exposición es reconocer que "La autoproducción de la ciudad se desarrolla, no por una acción cultural concertada y enriquecedora, como en la época pre-capitalista, sino como saldo de una lucha desigual de poderes" (ENET, 2008: 19). En el caso del AMGR, esta condición se plantea en términos de disputa permanente de acceso a la tierra, a la vivienda, a los servicios públicos (para unos pocos) y en definitiva, al territorio. El Estado "actúa y se retira" de escena, y las instituciones, los delegados, los vecinos son quienes trascienden dichas intervenciones; el reconocimiento y empoderamiento de capacidades de gestión y organización de los agentes locales es el desafío, pues son quienes continuarán operando en el territorio.

Por lo expuesto hasta aquí, la PSH se presenta de manera multiactoral, multisectorial, multi-dimensional y en permanente proceso de transformación. Por lo tanto, involucra la intervención de múltiples disciplinas, por lo que abordarla de manera integral implica atender a las diversas ramas del conocimiento y su ejercicio. En el siguiente apartado se explorará la incorporación en estos escenarios a la universidad como agente de acción y transformación. 


\section{Herramientas de "Retaguardia"}

Existe un avance en cuanto a reconocer las problemáticas del hábitat desde su complejidad, como elementos sistémicos y propuestas que tienden a pensarse como integrales, intersectoriales e interdisciplinarias, pero a la hora de materializarse cuentan con muchas carencias. Algunas discusiones al respecto son: niveles de participación, articulación de agentes y organismos, efectividad en el diagnóstico, entre otras. El desafío en relación con el hábitat es más amplio, es pensar más que en términos de una vivienda digna, en un desarrollo territorial integral.

DE SOUSA SANTOS (2012) retoma como modelo de trabajo la red denominada "conocimiento vivo" living knowledge 9 . Es en la actualidad uno de los casos paradigmáticos que, abordando los debates actuales aquí expuestos, se constituye en una propuesta en la cual la universidad confronta la injusticia cognitiva a través de la resignificación de sus funciones. La propuesta se presenta en un taller que integra aprendizaje, ciencia y desarrollo tecnológico vinculado con casos concretos del medio con distintos encuadres de trabajo desde la universidad. Esto implicaría un nuevo rol en la comunidad donde DE SANTOS SOUSA (2012) recomienda se produzca un espacio público de interconocimiento, reconociendo un nuevo paradigma de enseñaje, en el cual el que enseña aprende y el que aprende enseña. El autor atribuye el desplazamiento de la comunidad del entorno de la Universidad denunciando el alto grado de conocimiento científico, edificado con la convicción de verdad absoluta que denigra y silencia el conocimiento popular; esto se manifiesta en "los países periféricos, ricos en saberes no científicos y pobres en conocimiento científico, [que] transitan hacia este último bajo

9 Fuente: http://viewpointmag.com/2012/07/09/the-revolution-of-living-knowledge/\#container. la forma de la ciencia económica que destruye sus formas de sociabilidad, sus economías, sus comunidades indígenas y campesinas, y su medio ambiente" (DE SOUSA SANTOS, 2012: 172). El autor retoma lo actuado en Estados Unidos por equipos destinados a la "investigación comunitaria", que se organizan de manera global en una red, con una contundente reorientación de la universidad asociada de su desempeño. Dicha experiencia propone un "taller de ciencia" que vincule experiencias y problemáticas comunitarias con algunos de los tantos tipos de equipos que se constituyen en cátedras e institutos en cada facultad, lo que desde la perspectiva que plantea el autor se enriquecería significativamente las producciones (DE SOUSA SANTOS, 2012: 173).

\section{REFLEXIONES FINALES}

En términos de la promoción de un rol efectivo de la universidad, retomando las banderas de la reforma universitaria de 1918, que declaraba: "los dolores que nos quedan son las libertades que nos faltan", las deudas con la comunidad cobran un siniestro vínculo, si se toma conciencia de las deudas de transformación social de esta institución que el Estado argentino sostiene. En el devenir cotidiano de las prácticas de la Facultad de Arquitectura y Urbanismo de la UNNE que aportan a la Producción Social del Hábitat, trascienden escasos trabajos de impacto significativo en el medio donde se inserta esta unidad académica.

Los instituidos espacios de trabajos de vinculación con las problemáticas del hábitat en limitado números de cátedras, el número de participantes acotado en proyectos y el escaso reconocimiento de las actividades consideradas extracurriculares atentan con la participación de todos los claustros. Este escenario demanda la formación de nuevos encuadres de trabajos para dar respuestas efectivas 
tanto al reconocimiento institucional como al acto transformador al que debe volcarse la universidad a efectos de cumplir con su rol en la comunidad que la sostiene.

Es necesario combatir el enfoque mercantilista de la universidad, que vira cada vez más a la prestación de servicios a empresas y corporaciones políticas, y ponderar un espacio de diálogo con las urgentes demandas del medio a efectos de abordarlas y en su hacer promover escenarios de aprendizaje significativo tanto para estudiantes como para docentes, investigadores y graduados que perfeccionen la técnica para incidir de manera efectiva en un medio social como el nuestro que tanto lo requiere.

BIBLIOGRAFÍA

FERRERO, Aurelio (2013) Aprendizajes desde el hábitat popular: una experiencia pedagógica en la Universidad Nacional de Córdoba FAUD: 1991-2011. Aurelio Ferrero y Germán Gustavo Rebord; con prólogo de Enrique Ortiz Flores. 1.a ed., Córdoba: Asoc. Vivienda Económica (AVE), 128 pp. ISBN 978-987-27779-1-3.

MANFRED A. Max Neef (2004) Fundamentos de la Transdiciplinareidad. Universidad Austral de Chile, Agosto.

MAX NEEF, Manfred; ELIZALDE, Antonio y HOPENHAYN, Martín (1993). Desarrollo a escala humana. Ed. NORDAN Comunidad, Montevideo. PELLI, Víctor (2006) Habitar, participar, pertenecer. Acceder a la vivienda - incluirse en la sociedad. Buenos aires, Argentina, Nobuko.

PELLI, Víctor (1994) Clarificación y Replicabilidad. IIDVI. ICHOA.

DE SOUSA SANTOS, Boaventura (2012). "La Universidad en el Siglo XXI. Para una reforma democrática y emancipadora de la universidad Transformar la universidad para transformar la sociedad". En: Ramírez, René (ed.) La transformación de la universidad para transformar la sociedad. Quito: SENESCYT, 139-194.

DE SOUSA SANTOS, Boaventura (2011-12). "Introducción: las epistemologías del Sur". En: CIDOB (ed.) Formas-Otros. Saber, nombrar, narrar, hacer. Ediciones CIDOB, 9-22, Barcelona. 